\title{
Report of effective trametinib therapy in 2 children with progressive hypothalamic optic pathway pilocytic astrocytoma: documentation of volumetric response
}

\author{
Catherine Miller, MD, ${ }^{1}$ Daniel Guillaume, MD, ${ }^{1}$ Kathryn Dusenbery, MD, ${ }^{2}$ H. Brent Clark, MD, PhD, ${ }^{3}$ \\ and Christopher Moertel, MD4
}

Departments of ${ }^{1}$ Neurosurgery, ${ }^{2}$ Radiation Oncology, ${ }^{3}$ Pathology, and ${ }^{4}$ Pediatric Hematology/Oncology, University of Minnesota, Minneapolis, Minnesota

\begin{abstract}
Brain tumors are the most common solid tumor in childhood, and astrocytomas account for the largest proportion of these tumors. Increasing sophistication in genetic testing has allowed for the detection of specific mutations within tumor subtypes that may represent targets for individualized tumor treatment. The mitogen-activating protein kinase (MAPK) pathway and, more specifically, BRAF mutations have been shown to be prevalent in pediatric pilocytic astrocytomas and may represent one such area to target. Herein, the authors describe 2 cases of inoperable, chemotherapy-resistant pediatric pilocytic astrocytomas with a documented response to trametinib, an MAPK pathway inhibitor. While these cases were not treated in the setting of a clinical trial, their data support further ongoing clinical trial investigation to evaluate the safety and efficacy of this agent in pediatric low-grade gliomas.
\end{abstract}

https://thejns.org/doi/abs/10.3171/2016.9.PEDS16328

KEY WORDS pediatric; astrocytoma; MAPK pathway; BRAF mutation; trametinib; oncology

$\mathrm{B}$ RAIN tumors are the most common solid tumor in childhood, and astrocytomas account for the largest proportion of these tumors. The low-grade astrocytoma classification encompasses pilocytic astrocytomas (WHO Grade I), pilomyxoid astrocytomas (Grade II), infiltrating astrocytomas (Grade II), and pleomorphic xanthroastrocytoma (Grade I), and ganglioglioma (Grade I). Pilocytic astrocytomas are a group of heterogeneous tumors with various locations, radiological features, histological atypia, and clinical behavior. They can occur anywhere within the central nervous system (CNS); most are found in an infratentorial location. For some tumors, especially those in the hypothalamic and/or chiasmatic region, complete resection is impossible without significant deficit, and treatment must include adjuvant therapy given the less favorable progression-free and overall survival in patients with these lesions.

Within the past decade, significant breakthroughs have been made regarding the genetics of low-grade astrocytomas. Previously, few aberrations had been identified, but little was known regarding their tumorigenesis. The mitogen-activating protein kinase (MAPK) pathway transduces extracellular signals such as stress, hormones, cytokines, and growth factors to the nucleus via a series of consecutive phosphorylations. The result is a variety of physiological responses, including cell proliferation, differentiation, and apoptosis. Abnormalities in this pathway have been found in a high proportion (80\%) of pilocytic astrocytomas, with a smaller incidence (13\%) in Grade II tumors. ${ }^{9}$ Multiple mutations within $B R A F$ have been discovered, including the $B R A F \mathrm{~V} 600 \mathrm{E}$ point mutation and duplication of $B R A F$ at $7 \mathrm{q} 34 .^{2-4,6,11}$

Recently, multiple published articles have reported the $2-\mathrm{Mb}$ duplication of $7 \mathrm{q} 34$ causing the fusion of 2 genes. The N-terminus of KIAA1549 replaces the regulatory region of $B R A F$, resulting in a constitutively activated protein. The fusion product is expressed at higher levels than the wild-type $B R A F$ because the inhibitory domain that normally regulates $B R A F$ activity was lost. The deregulated $B R A F$ activity leads to increased downstream signaling (MEK/ERK) and subsequent increased cell proliferation. There are several other fusion variants that have been recognized; however, they are less common., 1,7,8,10

We present 2 cases of hypothalamic optic pathway pediatric astrocytomas that progressed despite multiple chemotherapy treatments. Each tumor was found to have 
duplication of $B R A F$ at $7 \mathrm{q} 34$ in the MAPK pathway and began treatment with a specific inhibitor-trametinib (Mekinist, GlaxoSmithKline, LLC). The patients' clinical course and response to the inhibitor are described in this report and summarized in Table 1.

\section{Methods \\ Patient Selection}

The medical records of all pediatric oncology patients treated by one of the senior authors were reviewed to identify patients with hypothalamic optic pathway low-grade astrocytomas that were progressive despite multiple chemotherapy regimens. Tumor pathology was verified, and then we identified those tumors that had undergone testing for $B R A F$ mutations. Two patients with tumors positive for $B R A F$ mutations at $7 \mathrm{q} 34$ were initiated on off-label trametinib therapy. Patients were followed up with serial examinations and imaging to determine clinical and radiological response, as well as any inhibitor-related adverse effects. This study was completed in accordance with a research protocol approved by the institutional review board at the University of Minnesota Medical Center. It followed the Health Insurance Portability and Accountability Act standards for privacy of personal health information.

\section{Quantification of Tumor Volumes}

Interval brain MRI was performed with a 3.0-T unit for each patient. Standard DICOM images of axial T2weighted MR images for Case 1 and postcontrast T1weighted MR images for Case 2 were transferred into a standard commercially available analysis software program (VelocityAI 3.1.0, Varian Medical Systems Inc.). Tumor volume was determined by manually selecting the tumor for each slice with 3D volume calculations made by the software and expressed in $\mathrm{cm}^{3}$. Measurements were made on baseline MR images obtained before initiating treatment and on subsequent MR images to determine treatment response.

\section{Statistical Analysis}

Four independent observers (senior neurosurgery resident, pediatric neurosurgeon, pediatric oncologist, and radiation oncologist) performed the measurements for each MR image at 2 different time points. Intraobserver reliability, the reproducibility of tumor volume by each observer, and interobserver reliability (overall agreement among the 4 observers) was estimated according to the method by Bland and Altman for intraclass and interclass correlation coefficients. The reliability estimates are shown with $95 \%$ confidence intervals. Statistical analysis was performed with the SPSS 13.0 statistical package (SPSS Inc.). Graphics were plotted with MedCalc 16.2 software (MedCalc).

\section{Case Reports}

\section{Case 1}

History and Examination

A 3-month-old female was noted to have congenital nystagmus, hyperopia, and decreased visual acuity. Magnetic resonance imaging showed a large multilobulated mass in the hypothalamic optic pathway region extending posteriorly to involve the hypothalamus and medial left temporal lobe (Fig. 1).

\section{Treatment}

Therapy with carboplatin and vincristine was initiated (according to Children's Oncology Group Protocol A9952 Regimen A). The child's tumor progressed with resultant obstructive hydrocephalus necessitating shunt placement and subtotal resection. Pathology was consistent with pilocytic astrocytoma (WHO Grade I; Fig. 2A). Bevacizu$\mathrm{mab}$ and irinotecan were started and subsequently withheld during a shunt revision. The patient was then treated

TABLE 1. Summary of characteristics in patients with pilocytic astrocytoma

\begin{tabular}{|c|c|c|}
\hline Parameter & Case 1 & Case 2 \\
\hline Sex & $\mathrm{F}$ & $\mathrm{F}$ \\
\hline Age at diagnosis (yrs) & 0.25 & 5.0 \\
\hline Tumor location & Optic pathway & Optic pathway \\
\hline NF1 & No & No \\
\hline Pathology & Pilocytic astrocytoma (WHO Grade I) & Pilocytic astrocytoma (WHO Grade I) \\
\hline Surgery & Subtotal resection $\times 2$ & Subtotal resection \\
\hline Prior chemotherapy & $V c+C ; B+I ; V c+C+T ; I+B ; V b$ & Vc + C; T; C; B + I; Tg + P + Vb; C + M \\
\hline Prior radiation & No & No \\
\hline BRAF status & Duplication of BRAF (7q34) & Duplication of BRAF (7q34) \\
\hline Time on medication (days) & 484 & 468 \\
\hline Tumor vol prior to treatment $\left(\mathrm{cm}^{3}\right)$ & 344.26 & 37.10 \\
\hline Tumor vol w/ treatment $\left(\mathrm{cm}^{3}\right)$ & 151.57 & 12.81 \\
\hline$\%$ decrease in vol & $55.9 \%$ & $65.5 \%$ \\
\hline Side effects & Yes & Yes \\
\hline Adverse events & No & No \\
\hline
\end{tabular}

$\mathrm{B}=$ bevacizumab; $\mathrm{C}=$ carboplatin $\mathrm{I}$ = irinotecan; $\mathrm{M}=$ melphalan; $\mathrm{NF1}=$ neurofibromatosis Type $1 ; \mathrm{P}=$ procarbazine; $\mathrm{T}=$ temozolomide; $\mathrm{Tg}=$ thioguanine; $\mathrm{Vc}=$ vincristine; $\mathrm{Vb}=$ vinblastine. 

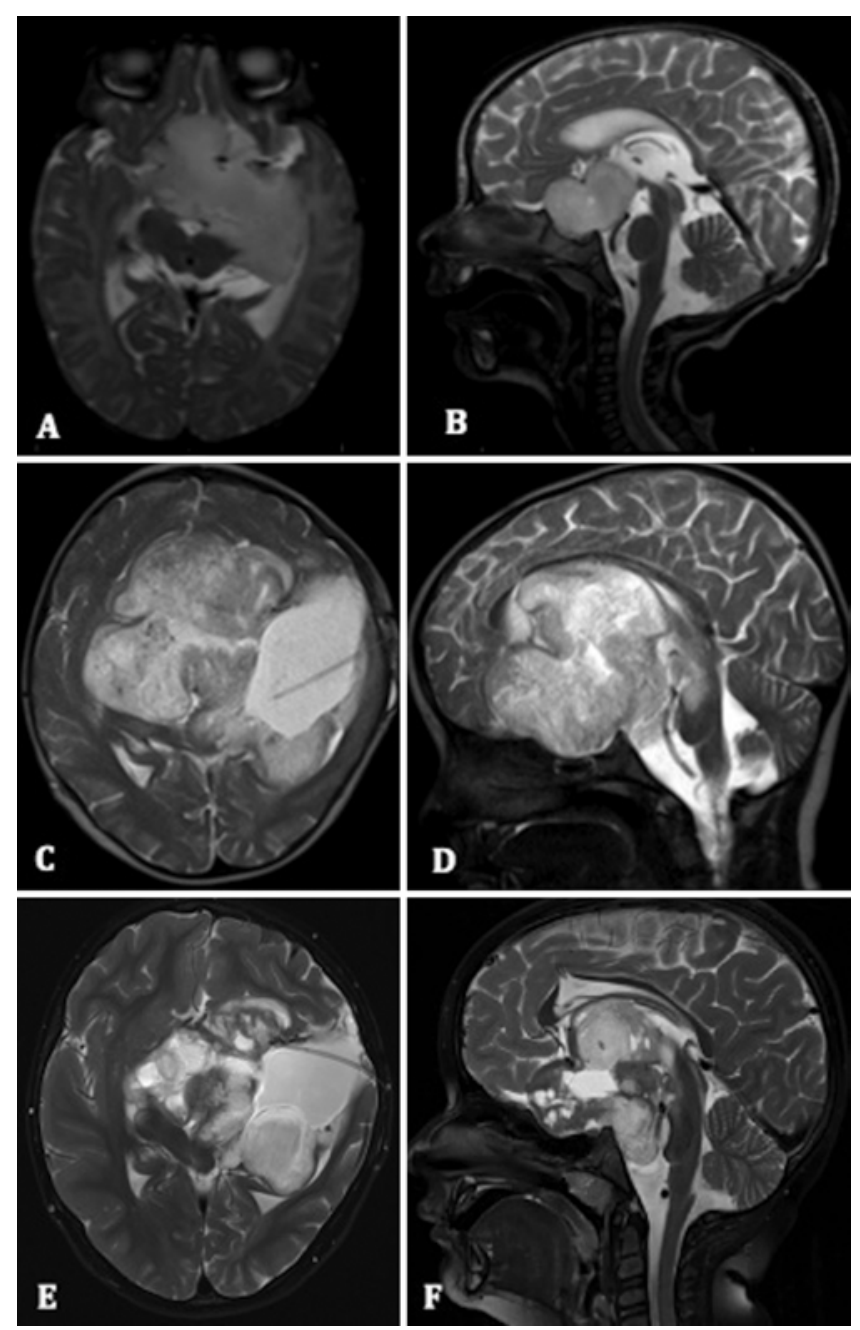

FIG. 1. Case 1. Initial axial (A) and sagittal (B) T2-weighted MR images showing the large heterogeneous midline lesion causing marked mass effect with a large cystic component. Axial (C) and sagittal (D) images obtained prior to trametinib therapy. Axial $(\mathbf{E})$ and sagittal $(\mathbf{F})$ images obtained after approximately 1.5 years of therapy, showing decreased tumor size and mass effect.

with vincristine, carboplatin, and temozolomide chemotherapy (according to Children's Oncology Group Protocol ACNS0223). Once that course was completed and the patient was off chemotherapy, the tumor significantly increased in size, necessitating recommencement of chemotherapy, this time with bevacizumab and irinotecan. Her tumor continued to grow despite this additional course, and thus vinblastine was tried. Despite alterations in therapy, her tumor continued to enlarge with progressive worsening of vision and an overall clinical decline. She underwent further tumor debulking, and $B R A F$ testing was performed on the obtained specimen. Her tumor was found to have a duplication of $B R A F$ (7q34). Given these findings and the child's worsening clinical status, we initiated trametinib $0.5 \mathrm{mg} / \mathrm{day}(0.035 \mathrm{mg} / \mathrm{kg})$ per gastrostomy tube.

\section{Subsequent Course}

The patient has had continued clinical improvement with serial MRI revealing tumor regression (Fig. 1E and

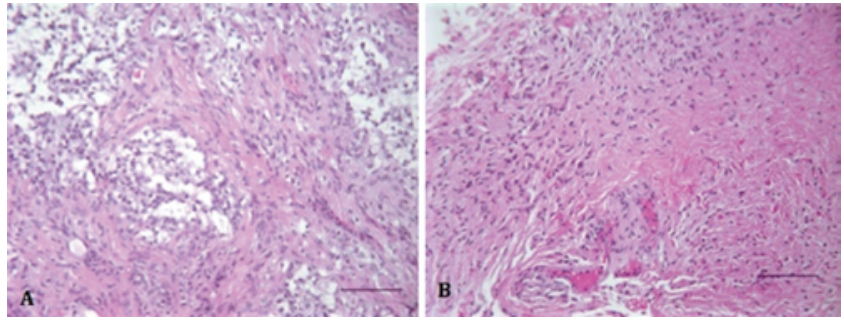

FIG. 2. Histopathological evaluation of the tumors was consistent with pilocytic astrocytoma in Case 1 (A) and Case 2 (B). Bar $=100 \mu \mathrm{m}$. Figure is available in color online only.

F). Ophthalmological examinations have shown an improvement from no vision in either eye prior to treatment to some ability to see color and shapes.

\section{Case 2}

History and Examination

At the age of 3 years, this girl was noted to have rightsided esotropia. Despite patching, the esotropia worsened and the patient developed headaches, proptosis, and papilledema of the right optic nerve. Imaging showed an enhancing lesion along the optic nerve anterior to the optic chiasm, consistent with an optic glioma (Fig. 3).

\section{Treatment}

A course of carboplatin and vincristine was completed. Approximately 1 year off of the chemotherapy, she had worsening visual acuity with progressive enlargement of the tumor. Separate courses of temozolomide then carboplatin were administered, but the tumor size continued to increase on both of these regimens. Therapy with bevacizumab and irinotecan stabilized the tumor. After 4 months off of chemotherapy, the patient was found to have significant tumor progression with new involvement of the bilateral optic nerve pathways adjacent to the optic chiasm. A second round of bevacizumab and irinotecan was completed. Again off of the chemotherapy, she had worsening vision and imaging showed tumor enlargement. She underwent debulking of the intracranial and intraorbital tumor, with pathology showing a pilocytic astrocytoma (WHO Grade I; Fig. 2B). BRAF testing was positive for duplication of BRAF (7q34). She completed 9 cycles of intraarterial chemotherapy (carboplatin and melphalan) with a stable tumor size. After the completion of intraarterial chemotherapy, she was started on trametinib $(1 \mathrm{mg}$ orally per day) with tumor decrease demonstrated on serial imaging (Fig. 3E and F).

\section{Subsequent Course}

Serial ophthalmological examinations showed stabilization of her visual fields and acuity in the affected eye after beginning treatment.

\section{Results}

\section{Tumor Volumetrics}

Volumes prior to inhibitor treatment were calculated to be $344.26 \mathrm{~cm}^{3}$ in Case 1 and $37.10 \mathrm{~cm}^{3}$ in Case 2. Both patients had a significant gradual decrease in tumor volume 

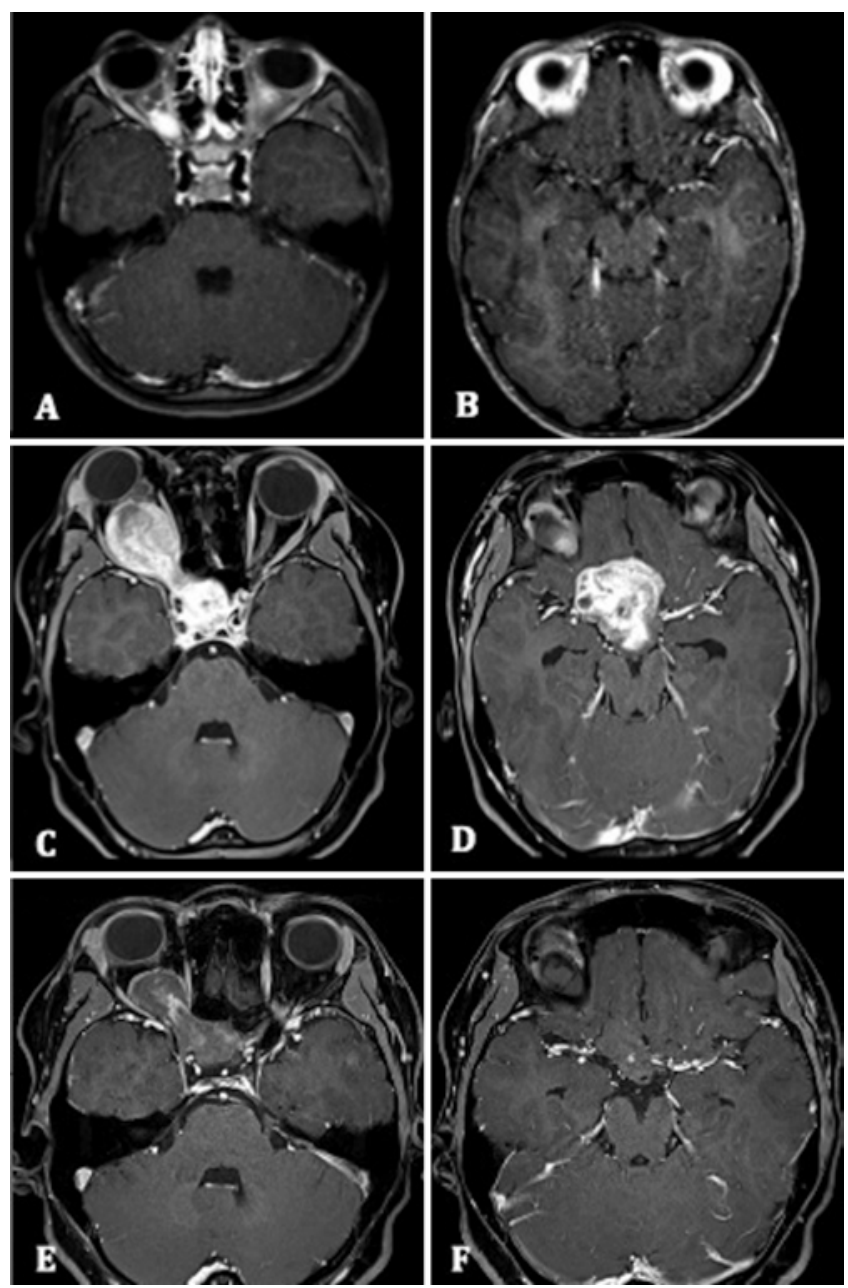

FIG. 3. Case 2. Initial axial T1-weighted MR images with contrast (A and $B$ ) showing a large lobulated mass in the right orbit extending to the optic canal with a large suprasellar cistern component. Axial MR images ( $C$ and $D$ ) obtained prior to trametinib therapy. Axial MR images ( $E$ and $F$ ) obtained after approximately 1.5 years of therapy, showing a decrease in tumor volume.

over time. On trametinib therapy the tumor volume in Case 1 decreased by $192.69 \mathrm{~cm}^{3}(55.9 \%)$, whereas that in Case 2 decreased by $24.29 \mathrm{~cm}^{3}$ (65.5\%; Fig. 4). The duration of treatment to date has been 484 and 468 days, respectively.

Both intraobserver and interobserver reliability were high for the measurements of tumor volume. The overall intraclass correlation coefficient was 0.9996 (95\% CI 0.9986-0.9999) and was slightly higher than the interclass correlation coefficient of 0.9985 (95\% CI 0.9957-0.9996). For the individual patients, the intraclass correlation coefficient ranged from 0.9976 to 0.9964 (95\% CI 0.9870 $0.9995,95 \%$ CI $0.9745-0.996$, respectively), while the interclass correlation coefficient varied from 0.9494 to 0.9895 (95\% CI $0.7378-0.9965,95 \%$ CI $0.9580-0.9984$, respectively).

\section{Discussion}

Each of our patients had a gradual decline in tumor volume with concurrent improvement in clinical symptoms.

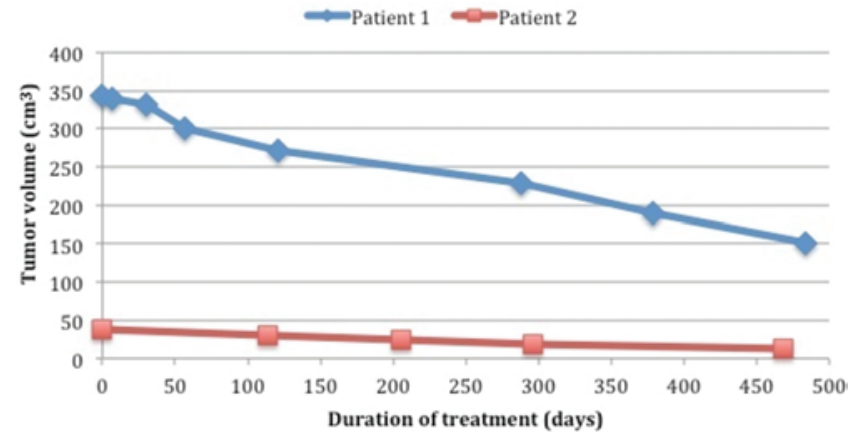

FIG. 4. Graphic representation for each patient showing gradual decrease in tumor volume over time. Figure is available in color online only.

Figure 4 shows the decrease in volume over the study period with no evidence of a plateau. Both tumors decreased more than $50 \%$ with the inhibitor. The Velocity system allowed easy measurement and comparison between serial images to ensure completeness in the volumetrics. Statistical analysis revealed that tumor volumes measured with this system were reliable even among the different observers. This was true despite the fact that our observers had different specialties and levels of training.

Pilocytic astrocytomas are sometimes characterized as a chronic disease as the 10-year survival is greater than $95 \%$. Gross-total resection is reported in $94 \%$ of cerebellar tumors but only $3.2 \%$ of hypothalamic and/or chiasmatic tumors. The rate of progression ranges from $50 \%$ to $80 \%$ in tumors for which gross-total resection is not achievable. Given the location of each of our patient's tumors, complete resection was impossible, thus necessitating the use of chemotherapy and/or radiation therapy. Both tumors progressed despite "standard-of-care" adjuvant therapy, which prompted us to investigate possible alternative therapies. Increasing sophistication in genetic testing has allowed for the detection of specific mutations within tumor subtypes that could represent targets for individualized tumor treatment. The MAPK pathway and, more specifically, $B R A F$ mutations have been shown to be prevalent in pediatric astrocytomas and could represent important areas to target. Pathways identified in tumorigenesis are the targets for many inhibiting or altering agents. However, as the MAPK pathway is involved in development, inhibition of this pathway could lead to widespread unintended results. Therefore, identifying and targeting specific anomalies in the pathway have become goals in treating tumors with these mutations.

Multiple clinical trials investigating targeted molecular agents for tumors have been completed or launched in an effort to offer individualized patient treatment. Many of the MAPK inhibitors have been studied in neoplasms outside the CNS. Trametinib, an MEK1 and MEK2 kinase inhibitor, is thought to inhibit proliferation but not induce apoptosis. It was approved by the Food and Drug Administration in 2014 for use in $B R A F$ V600E-mutated metastatic melanoma after showing improved survival both alone and in combination with dabrafenib (Tafinlar, GlaxoSmithKline, LLC), a BRAF inhibitor similar to vemurafenib (Zelboraf, Hoffmann-LaRoche Inc.). One phase I/IIa clinical trial (NCT02124772, clinicaltrials.gov) 
is studying its effects in refractory or recurrent pediatric tumors of all origins. One arm of the trial is specifically investigating effects on low-grade gliomas. Both of our patients were started on trametinib prior to commencement of this study. The patient in Case 1 would have been excluded from the trial given her poor Karnofsky Performance Status prior to beginning treatment. Trametinib was prescribed off-label in each of our patients.

There are no published reports of trametinib use in pediatric low-grade gliomas, and to our knowledge our patients are the first 2 reported cases of documented tumor regression in $B R A F$-mutated pediatric gliomas. We acknowledge that our study was not performed as part of a prospective clinical trial; therefore, our results are insufficient to support the safety and efficacy of these agents. Effective evaluation of MEK inhibitors will ultimately require appropriate prospective clinical trial investigation, and work is being done toward this goal. Our data suggest that an ongoing trial may have positive results in patients with low-grade gliomas, despite their anecdotal nature.

One concern with the use of specific inhibitors is the development of resistance. Many patients who initially respond to treatment with RAF or MEK inhibitors have disease progression consistent with inhibitor resistance. This has been shown in several clinical trials, with progression reported from 2 to 18 months after the start of the inhibitor. ${ }^{5}$ Several mechanisms account for this resistance, including additional mutations in the target molecule and compensatory activation of the pathway at upstream or downstream locations. Studies are currently underway, investigating the effect of combinations of inhibitors, which affect multiple targets or pathways. ${ }^{6}$ To date, neither of our patients has developed resistance to the inhibitor, although each has been treated for less than 18 months and is within the described period of risk for developing resistance. However, given the anticipated duration of treatment, it is possible that this resistance may occur at some point in their treatment course. We plan to continue the trametinib until there is disease progression or the development of adverse events from toxicity, thus necessitating long-term monitoring of our patients.

As with any treatment, there are side effects with the potential for severe adverse reactions. Trametinib has been associated with cutaneous malignancy, hemorrhage or thromboembolism, cardiomyopathy, ocular toxicity, and serious skin toxicity. The most common ( $>20 \%)$ adverse events noted with trametinib are rash, diarrhea, and alopecia. Both of our patients developed mild rashes that were treated with topical agents and oral antibiotics. No severe adverse effects were noted in either of them. Overall, these patients have experienced an excellent quality of life.

Effective evaluation of tumor response to a treatment is necessary, and tumor volume is an important metric to be considered to determine if a therapy is effective. Assessment of the radiological response can be arduous, especially when tumors have been resected, are cystic, or have significant intratumoral hemorrhage or peritumoral edema. Additional difficulties may arise when there is no clear plane between the tumor and normal brain parenchyma or if there is variation in slice acquisition or contrast dose timing. To address these issues, we chose images in which the tumor was clearly delineated from normal tissue and used the same image series for each measurement and comparison. We also confirmed the same slice thickness for each image to ensure consistency.

New volumetric techniques have been developed to facilitate these measurements; however, concerns remain regarding the variability between single and multiple observers. In our study, the intra- and interclass correlation coefficients were very high, showing excellent reliability in measuring the tumor volumes.

\section{Conclusions}

We have shown in 2 patients that trametinib therapy for tumors with $B R A F$ mutations is effective in decreasing tumor volume in otherwise progressive pediatric hypothalamic optic pathway astrocytoma. Our results suggest that these mutations may be ideal targets for the treatment of tumors and that tumor volume measurement is a reasonable method for documenting response in this patient group. While these cases were not treated in the setting of a clinical trial, our data support further ongoing clinical trial investigation to evaluate the safety and efficacy of this agent in pediatric low-grade gliomas.

\section{References}

1. Chen YH, Gutmann DH: The molecular and cell biology of pediatric low-grade gliomas. Oncogene 33:2019-2026, 2014

2. Collins VP, Jones DT, Giannini C: Pilocytic astrocytoma: pathology, molecular mechanisms and markers. Acta Neuropathol 129:775-788, 2015

3. Jacob K, Albrecht S, Sollier C, Faury D, Sader E, Montpetit A, et al: Duplication of 7q34 is specific to juvenile pilocytic astrocytomas and a hallmark of cerebellar and optic pathway tumours. Br J Cancer 101:722-733, 2009

4. Jones DT, Gronych J, Lichter P, Witt O, Pfister SM: MAPK pathway activation in pilocytic astrocytoma. Cell Mol Life Sci 69:1799-1811, 2012

5. Karajannis MA, Legault G, Fisher MJ, Milla SS, Cohen KJ, Wisoff JH, et al: Phase II study of sorafenib in children with recurrent or progressive low-grade astrocytomas. Neuro Oncol 16:1408-1416, 2014

6. McCubrey JA, Steelman LS, Chappell WH, Abrams SL, Franklin RA, Montalto G, et al: Ras/Raf/MEK/ERK and $\mathrm{PI} 3 \mathrm{~K} / \mathrm{PTEN} / \mathrm{Akt} / \mathrm{mTOR}$ cascade inhibitors: how mutations can result in therapy resistance and how to overcome resistance. Oncotarget 3:1068-1111, 2012

7. Penman CL, Faulkner C, Lowis SP, Kurian KM: Current understanding of BRAF alterations in diagnosis, prognosis, and therapeutic targeting in pediatric low-grade gliomas. Front Oncol 5:54, 2015

8. Roth JJ, Santi M, Pollock AN, Harding BN, Rorke-Adams LB, Tooke LS, et al: Chromosome band 7q34 deletions resulting in KIAA1549-BRAF and FAM131B-BRAF fusions in pediatric low-grade gliomas. Brain Pathol 25:182-192, 2015

9. Schindler G, Capper D, Meyer J, Janzarik W, Omran H, Herold-Mende C, et al: Analysis of BRAF V600E mutation in 1,320 nervous system tumors reveals high mutation frequencies in pleomorphic xanthoastrocytoma, ganglioglioma and extra-cerebellar pilocytic astrocytoma. Acta Neuropathol 121:397-405, 2011

10. Shin CH, Grossmann AH, Holmen SL, Robinson JP: The BRAF kinase domain promotes the development of gliomas in vivo. Genes Cancer 6:9-18, 2015

11. Tatevossian RG, Lawson AR, Forshew T, Hindley GF, El- 
lison DW, Sheer D: MAPK pathway activation and the origins of pediatric low-grade astrocytomas. J Cell Physiol 222:509-514, 2010

\section{Disclosures}

The authors report no conflict of interest concerning the materials or methods used in this study or the findings specified in this paper.

\section{Author Contributions}

Conception and design: Miller, Guillaume, Moertel. Acquisition of data: Miller. Analysis and interpretation of data: all authors. Drafting the article: Miller. Critically revising the article: Miller,
Guillaume, Moertel. Reviewed submitted version of manuscript: Miller, Guillaume, Dusenbery, Moertel. Approved the final version of the manuscript on behalf of all authors: Miller. Statistical analysis: Miller.

\section{Supplemental Information}

\section{Previous Presentations}

This work was previously presented in digital poster and abstract form at the 44th Annual AANS/CNS Section on Pediatric Neurological Surgery held in Seattle, Washington, on December 8-11, 2015.

\section{Correspondence}

Catherine Miller, Department of Neurosurgery, University of Minnesota, 420 Delaware St. SE, MMC 96, Rm. D429, Mayo Building, Minneapolis, MN 55455. email: mill5459@umn.edu. 